\title{
Engineering surface waves in flat phononic plates
}

\author{
Héctor Estrada, ${ }^{1,2, *}$ Pilar Candelas, ${ }^{1}$ Francisco Belmar, ${ }^{1}$ Antonio Uris, ${ }^{1}$ \\ F. Javier García de Abajo, ${ }^{3}$ and Francisco Meseguer ${ }^{1,2, \dagger}$ \\ ${ }^{1}$ Centro de Tecnologías Físicas, Unidad Asociada ICMM-CSIC/UPV, Universidad Politécnica de Valencia, \\ Av. de los Naranjos s/n. 46022 Valencia, Spain \\ ${ }^{2}$ Instituto de Ciencia de Materiales de Madrid (CSIC), Cantoblanco, 28049 Madrid, Spain \\ ${ }^{3}$ IQFR-CSIC, Serrano 119, 28006 Madrid, Spain
}

(Received 21 December 2011; revised manuscript received 2 March 2012; published 1 May 2012)

\begin{abstract}
Surface acoustic-wave phenomena span a wide range of length scales going from the devastation of earthquakes down to image reconstruction of buried nanostructures. In solid-fluid systems, the so-called Scholte-Stoneley waves (SSWs) dominate the scene at the interface with their evanescent fields decaying away into both media. Understanding and manipulating these waves in patterned surfaces would enable new applications of sound to be devised for imaging and acoustic signal processing, although this task has so far remained elusive. Here, we report SSW modes displaying directional gaps and band folding in fluid-loaded solid phononic plates. The plates are inhomogeneously patterned with in-plane periodic modulations of the elastic constants, but present flat surfaces free of corrugations. We experimentally demonstrate control of SSWs, which opens a promising route toward acoustic fluid sensing, microscopy, and signal processing.
\end{abstract}

DOI: 10.1103/PhysRevB.85.174301

PACS number(s): 43.35.+d, 43.20.Fn, 42.79.Dj, 43.30.Jx

\section{INTRODUCTION}

Funnelling waves through small apertures perforated in opaque plates has served as a source of inspiration in optics and acoustics alike, leading to remarkable applications such as enhanced optical sensing in hole arrays, ${ }^{1}$ perfect light absorption, ${ }^{2}$ and extraordinary sound screening. ${ }^{3}$ In this context, extraordinary optical transmission ${ }^{4}$ has stimulated a large deal of work intended to extrapolate the physics and understanding of light interaction with perforated plates to acoustics. However, the mechanisms involved in sound transmission through subwavelength apertures have distinct features that prove the photonic analogy to be misleading, as emphasized by the following established results: (i) at variance with optics, sound transmission through subwavelength holes is not extraordinary at all;, 5 (ii) the interaction with guided elastic modes becomes critical in assessing the effect of periodic corrugation induced by apertures in acoustic hole arrays $;^{7}$ and (iii) the surrounding medium (e.g., air) only produces circumstantial effects in the interaction of light with a good metal, but it plays a pivotal role in acoustics.

Surface (Rayleigh) ${ }^{8}$ and guided-plate (Lamb) waves $^{9}$ are known to be trapped by a solid that is held in vacuum. However, if the solid is surrounded by a fluid, both Rayleigh and Lamb waves become leaky and interface modes known as Scholte-Stoneley waves (SSWs) emerge. ${ }^{10}$ Additionally, a periodic modulation imprinted on a plate induces extra surface modes, ${ }^{11-13}$ whose characteristics are independent of the solid properties as long as the solid-fluid acoustic impedance mismatch remains high (e.g., in air-solid systems). Moreover, surface modes strongly hybridize with Fabry-Perot resonances of the hole cavities in drilled plates. ${ }^{13}$ These concepts have spurred a rich literature encompassing resonant acoustic transmission through periodically perforated plates, ${ }^{3,14-16}$ corrugation-assisted sound collimation, ${ }^{17}$ backward beam displacement, ${ }^{18,19}$ and hyperlensing. ${ }^{20}$ However, driven by the optics interpretation, perforated plates with subwavelength holes have been studied from different mutually exclusive perspectives, namely, either as sound barriers with waves evolving just in the fluid ${ }^{14-16}$ or as elastic phononic crystals in which sound propagates along the plate. ${ }^{21,22}$ Actually, interface modes in phononic plates (PPs) have not yet been modelled or measured, and only very preliminary experimental results have been reported on SSWs supported by singly and doubly corrugated phononic crystals on a surface. ${ }^{23}$

Here, we demonstrate the dominant role of interface and Lamb modes in periodically patterned flat solid plates. We present a comprehensive study of the dispersion relation of these modes in plates consisting of homogeneous solid films periodically patterned with arrays of cylindrical inclusions made of a different solid material. Two complementary experimental techniques allow us to detect all modes above and below the sound line. We report measurements that are well understood from a full elastoacoustic model providing a complete description of radiating and non-radiating SSW modes trapped at the fluid-plate interface. Our control of SSWs is based on mechanisms differing from diffraction and Fabry-Perot resonances typical of pierced plates, and notably, the degree of confinement of trapped SSW is much larger in the samples here presented.

\section{RESULTS AND DISCUSSION}

Throughout this article, we study plate modes and their dispersion relations in the space of frequency $\omega$ and parallel wave vector $\mathbf{k}_{\|}$[see Fig. 1(a) for a scheme of a PP]. For simplicity, only variations of $\mathbf{k}_{\|}$along the $\Gamma \mathrm{X}$ direction of the first Brillouin zone [see Fig. 1(a), inset] are considered. Two transmission regimes can be distinguished depending on the irradiation conditions. For an incident propagating wave, characterized by $k_{\|}<\omega / c_{0}$, where $c_{0}$ is the sound velocity in the fluid, the $\left(\mathbf{k}_{\|}, \omega\right)$ dependence of the transmission $\tau$ yields information of the plate-fluid coupling and the presence of leaky surface modes. ${ }^{7}$ In contrast, incident evanescent waves $\left(k_{\|}>\right.$ $\omega / c_{0}$ ) provide information about nonleaky bound modes.

We present underwater sound transmission measurements ${ }^{7}$ in Figs. 1(b) and 1(d) for two types of PPs, namely, aluminum 
(a)
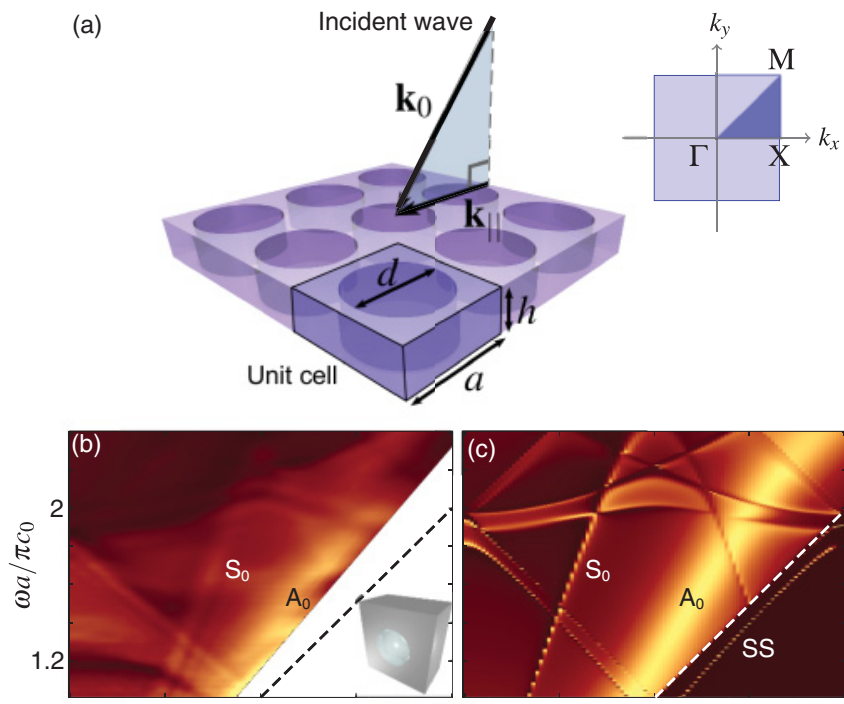

$\frac{\stackrel{\Xi}{~}}{3}^{2}$

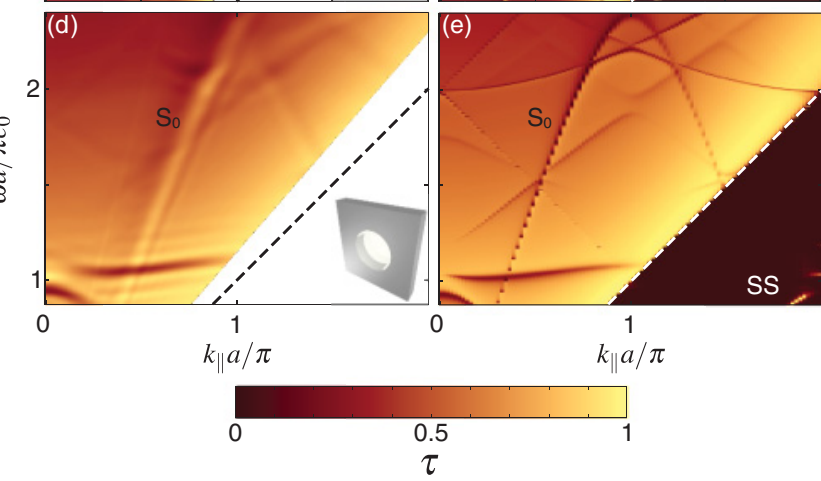

FIG. 1. (Color online) (a) Scheme of a PP, its geometrical parameters, and the orientation of an incident sound wave. The host plate of thickness $h$ is periodically decorated with cylindrical inclusions of diameter $d$ arranged in a square lattice of period $a$. The incident wave, represented by its wave vector $\mathbf{k}_{0}$, determines the parallel wave-vector component $\mathbf{k}_{\|}$. The inset shows the first Brillouin zone. Measured [(b), (d)] and calculated [(c), (e)] fraction $\tau$ of transmitted sound power (color scale) as a function of normalized parallel wave vector $k_{\|} a / \pi$ and frequency $\omega a / \pi c_{0}$. Results for an aluminum-PMMA PP of dimensions $d=3 \mathrm{~mm}, a=5 \mathrm{~mm}$, and $h=3 \mathrm{~mm}$ [see inset in (b)] are shown in panels (b) and (c). Panels (d) and (e) correspond to an aluminum-epoxy PP having $d=2.5 \mathrm{~mm}$, $a=5 \mathrm{~mm}$, and $h=0.85 \mathrm{~mm}$ [see inset in (d)]. The sound line (dashed) is indicated in all cases.

with poly-methyl metacrylate (PMMA) inclusions [see Fig. 1(b)] and aluminum with epoxy resin inclusions [see Fig. 1(d)]. Our angle-resolved measurements cover a limited range of incidence angles $\left(0^{\circ}-60^{\circ}\right)$ within the sound cone, which are accessed by tilting the sample around the direction of a nearest-neighbor axis of the inclusion lattice. For the aluminum-PMMA PP [see Fig. 1(b)], zero-order Lamb modes are clearly distinguishable. The $\mathrm{A}_{0}$ mode couples to the surrounding fluid producing a broad peak featuring full transmission. In contrast, the $\mathrm{S}_{0}$ mode preserves its less radiative nature, showing up as a very sharp feature. These results are in excellent agreement with the simulations shown in Fig. 1(c), which are based upon a full elastoacoustic model ${ }^{7,24}$ (FEAM) that incorporates the elastic properties of the structured plate. The periodic band structure is clearly emphasized by mode doubling beyond $k_{\|}>\pi / a$, particularly, in features resembling Wood anomaly minima. ${ }^{7}$ Negative group velocity and band maxima associated with zero group velocity are clearly resolved in both theory and experiment. Incidentally, there is a measured transmission minimum at low frequencies that is not predicted by theory, presumably arising from PP imperfections.

Calculations of the transmission of solid-solid PPs have been performed using an implementation of the FEAM described elsewhere. ${ }^{7,24}$ In the FEAM, the two-dimensional solid-solid problem is solved using plane-wave expansions for both the elastic constants and the displacement in the translationally invariant crystal with the same symmetry [a square lattice of period $a$, see Fig. 1(a)] and cylindrical inclusions (diameter $d$ ) as the original plate. The fields in the plate are then expanded in terms of guided modes by fixing $\left(\mathbf{k}_{\|}, \omega\right)$ and solving a quadratic diagonalization problem in which the wave vector component parallel to the cylinders $\left(k_{z}\right)$ is the eigenvalue. We further use a Rayleigh expansion to describe the wave field in the fluid (incident and transmitted waves, along with their diffracted beams). The continuity of the displacement $\mathbf{u}$ and normal stress $\sigma_{i z}$ is then imposed for a finite plate of thickness $h$. As the fluid only supports longitudinal vibrations, its stress tensor reduces to a scalar pressure $\left(\sigma_{i j}=\right.$ $-p \delta_{i j}$ ). Viscosity effects are neglected. The transmitted sound power $\tau$ is finally calculated for any given frequency $\omega$ and parallel wave vector $\mathbf{k}_{\|}$from the self-consistent coefficients of the noted Rayleigh expansions. The elastic constants of the different materials used in the calculations (density $\rho$ as well as longitudinal and transversal sound velocities, $c_{\ell}$ and $c_{t}$ ) are detailed in Ref. 25.

The measured transmission dispersion of the aluminumepoxy PP [see Fig. 1(d)] looks very different from that of aluminum-PMMA not only because of the different geometrical parameters $(d=2.5 \mathrm{~mm}, a=5 \mathrm{~mm}$, and $h=0.85 \mathrm{~mm})$, but also because of the higher attenuation produced by the epoxy resin, which has been incorporated in the corresponding calculations [see Fig. 1(e)] and leads to smoother features. Wood anomalies are too narrow to be observed in experiment and do not play the key role that they have in perforated plates. ${ }^{7}$ Interestingly, the aluminum-epoxy PP presents a nearly flat band at low frequencies both in theory and experiment. In addition, the $\mathrm{S}_{0}$ mode is clearly observable, including its mirror in the second Brillouin zone, which features negative group velocity. However, the $\mathrm{A}_{0}$ mode is difficult to resolve in the transmission for this frequency range and plate thickness, because it is too broad and overshadowed by the overall high transmission observed near the sound line. Finally, a bound SSW is predicted by theory below the sound cone, which has a replica at small $k_{\|}$that is clearly discernible in experiment. Unfortunately, the transmission measurements do not grant us access to this bound SSW, and thus we use a complementary near-field setup to that end.

Our near-field in-plate excitation measurement (NiPEM) setup is schematically represented in Fig. 2(a). A needle hydrophone (Precision Acoustics, $1 \mathrm{~mm}$ wide) is used as the receiver and a small piezoelectric disk (Steiner \& Martins Inc. model SMD15T09S411, $15 \mathrm{~mm}$ in diameter and $1 \mathrm{~mm}$ thick) is attached to the plate and employed as a source [see Fig. 2(a)]. The piezoelectric actuator is bonded to the plate by means of 

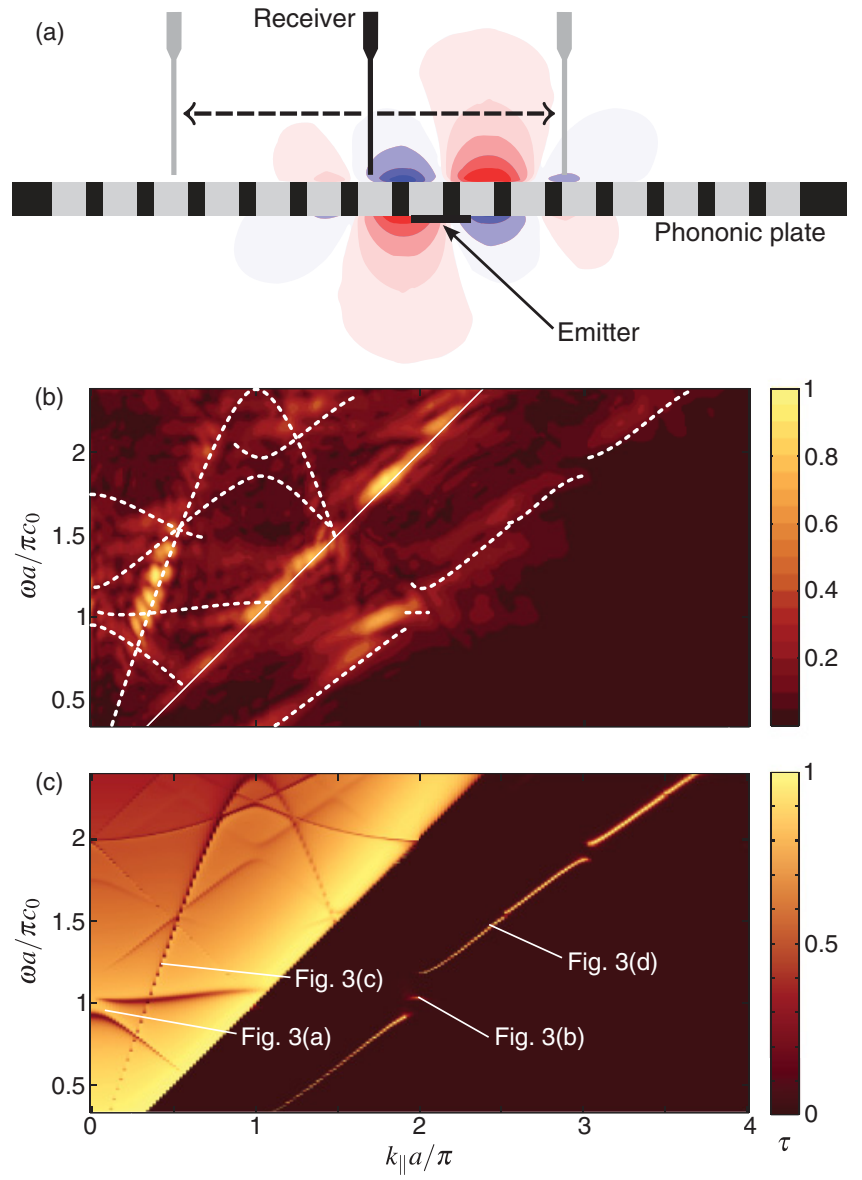

FIG. 2. (Color online) (a) Scheme of our near-field measurement technique. (b) Near-field dispersion measurement for the PP of Fig. 1(d), with the color scale showing the normalized twodimensional Fourier transform of the space-time resolved measured amplitude. White dashed curves show the modes predicted by FEAM calculations. (c) Transmitted sound power $\tau$ as a function of normalized parallel wave vector $k_{\|} a / \pi$ and frequency $\omega a / \pi c_{0}$ from FEAM calculations for an underwater aluminum-epoxy PP. Wave fields at the points marked by labels in the dispersion curves are shown in Fig. 3. The PP geometrical parameters are $d=2.5 \mathrm{~mm}$, $a=5 \mathrm{~mm}$, and $h=0.85 \mathrm{~mm}$.

cyanoacrylate based adhesive and covered with a thin silicone layer to avoid electrical short circuit. The dispersion of the plate modes is then measured by performing a linear spatial scan with the hydrophone on the inspected plate surface, taking into account the array symmetry when choosing the scanning path. A total scanned length of $220 \mathrm{~mm}$, covering the whole array in steps of $0.25 \mathrm{~mm}$ and at $\sim 1 \mathrm{~mm}$ from the plate surface is measured using an automated positioning system.

Common techniques use liquid ${ }^{26}$ and solid ${ }^{27}$ wedges attached to the transducers to excite Lamb waves. However, considering the geometry of the array, a simple piezoceramic disk at its center is found to be more appropriate to our purposes.

This experimental setup renders a position- and timedependent amplitude, which we Fourier transform ${ }^{28}$ to obtain an amplitude in $\left(\mathbf{k}_{\|}, \omega\right)$ space. The result is shown in Fig. 2(b) for the same underwater aluminum-epoxy PP as in Figs. 1(d) and 1(e). The normalized Fourier transform depicted in
Fig. 2(b) is an average of data acquired for both negative and positive $k_{\|}$along a $\Gamma \mathrm{X}$ direction, to minimize the effect of small deviations in the position of the piezoelectric source with respect to the array. The corresponding FEAM calculations are shown in Fig. 2(c), from which we extract dispersion mode features that are superimposed on Fig. 2(b) as dashed curves. The $\mathrm{A}_{0}$ feature that was difficult to identify in transmission is now clearly resolved in the NiPEM dispersion right at the sound line (solid white line). There is a clear correlation between measured maxima and FEAM calculated modes, which corroborates the suitability of our NiPEM technique to resolve dispersion relations, particularly below the sound cone, which was inaccessible to transmission measurements. In particular, we observe nonleaky modes exhibiting band gaps and regions of negative group velocity, which have not been reported so far.

The relation of these nonleaky modes to SSWs can be demonstrated by comparing the $c_{t} / c_{0}$ ratios between the lowest transversal sound velocity in the solids forming the PP and the nominal speed of sound in water $c_{0}=1480 \mathrm{~m} / \mathrm{s}$. For the aluminum-PMMA PP, this ratio reaches 0.97 , whereas
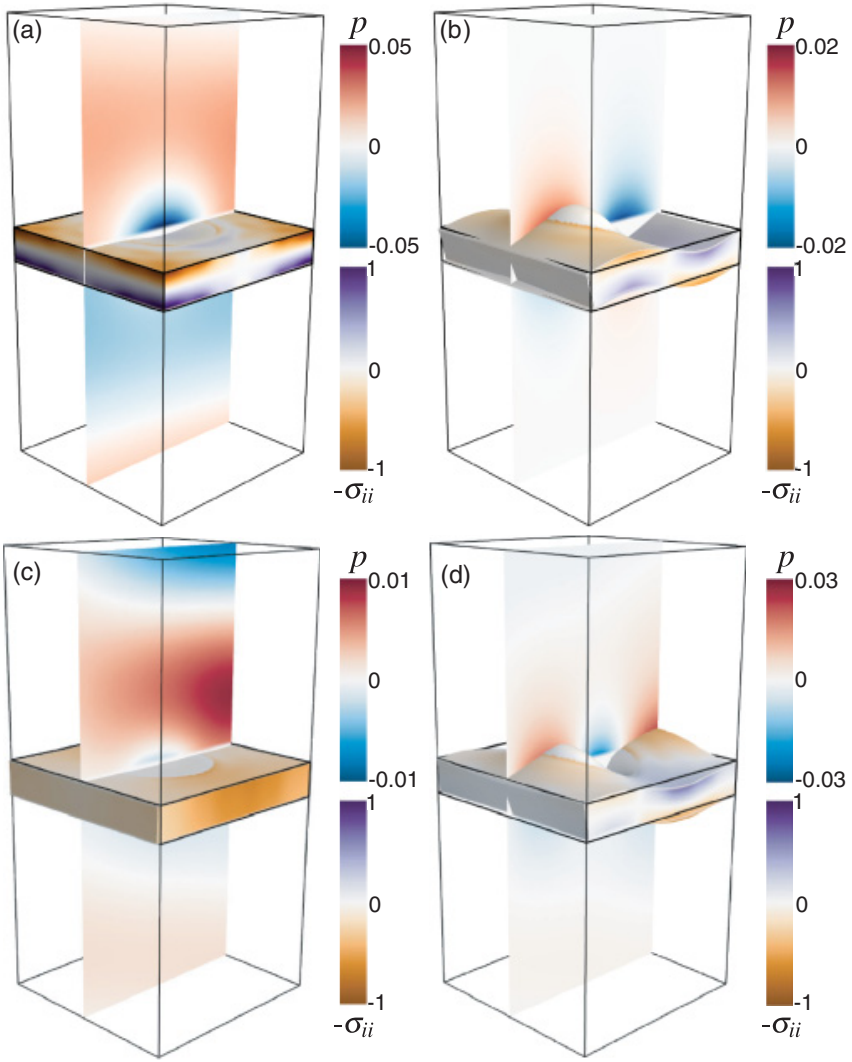

FIG. 3. (Color online) The plots show the fluid pressure $p$ (red-blue scale), the normalized first principal stress $\left(-\sigma_{i i}\right.$ in the $\mathrm{PP}$, purple-orange scale), and the displacement vector (plate surface deformation) within a unit cell for selected values of $\left(\mathbf{k}_{\|}, \omega\right)$, as indicated in Fig. 2(c). Two leaky [(a) and (c)] and two nonleaky [(b) and (d)] modes are considered. The incident wave comes from the upper right side of the plots [see Fig. 1(a)]. The epoxy cylinder is placed in the center of the unit cell. The plate deformation is exaggerated for clarity, but its relative amplitude is maintained in all four modes. 
for the aluminum-epoxy PP it is 0.64. Comparing now Figs. 1(c) and 1(e), we can clearly relate the separation of the modes from the sound line for a given frequency to the $c_{t} / c_{0}$ ratio, which is consistent with observations of wave propagation in fluid-solid interfaces. ${ }^{29}$ A fluid-solid interface exhibits Rayleigh waves if $c_{t}>c_{0}$, but it is only governed by SSWs if $c_{t}<c_{0}$. In our samples, because we are dealing with PPs that contain two different materials, this condition is reflected in the distance from the nonleaky modes to the sound line, which is larger for epoxy than for PMMA inclusions and is far larger than that achieved by means of corrugations. ${ }^{13}$

We obtain further insight into guided modes by analyzing their field distributions in the fluid and in the PP. Figure 3 shows calculated distributions within a plate unit cell for four different points of the dispersion diagram of Fig. 2(c) (see labels). Nearfield plots are obtained by explicitly evaluating both Rayleigh and plane-wave expansions of the FEAM. Specifically, we represent the first principal stress $-\sigma_{i i}=-\left(\sigma_{x x}+\sigma_{y y}+\sigma_{z z}\right)$ and the displacement $\mathbf{u}$ in the PP, as well as the pressure $p$ in the fluid. The orientation of the wave fields follows the diagram of Fig. 1(a), with the incident wave coming from the upper part for excitation of leaky modes. The first point of interest [see Fig. 3(a)] corresponds to the first folded SSW mode at $\left(k_{\|} a / \pi, \omega a / \pi c_{0}\right)=(0.08,0.94)$. The negative pressure maximum near the plate in the epoxy cylinder is caused by its strong deformation, compensated by the lower deformation of the aluminum and its higher stress. The incident wave is almost fully transmitted through the plate (from top to bottom), whereas the plate vibration has a relatively low displacement amplitude and negative group velocity (see video 1 in Ref. 31). We then focus on a nonleaky SSW mode at $(1.98,1.04)$ with zero group velocity and a standing-wave character (see video 2 in Ref. 31), which we plot in Fig. 3(b). The PP displacement clearly shows two maxima producing evanescent waves in the fluid. This is in contrast to the long wavelength (larger than the unit cell) traveling $\mathrm{S}_{0}$ mode at $(0.42,1.23)$ [see Fig. 3(c)]. The incident plane wave couples the Lamb mode and it reradiates a wave to both fluid half-spaces, so that the top pressure field is the sum of the incident and the reflected field (see video 3 in Ref. 31). On the nonleaky side at $(2.42,1.47)$, Fig. 3(d) reveals a field similar to that of Fig. 3(b) (i.e., several displacement maxima exist in the plate and the pressure field is evanescent in the fluid). However, this is a traveling SSW, as inferred from its position in the dispersion diagram (see video 4 in Ref. 31).

\section{CONCLUSIONS}

In summary, the behavior of the phononic plates here studied differs from common perforated plates or corrugated surfaces, in particular due to (i) the absence of Fabry-Perot resonances, (ii) the strong effect that the inclusions have on intrinsic modes of the plate, leading to a varied band structure encompassing mode mixing and band folding to unprecedented levels, and (iii) the presence of textured trapped SSW modes outside the sound cone exhibiting zero and negative group velocity at some points in the dispersion diagram. We experimentally demonstrate control of SSWs, fully supported by theoretical calculations. Our use of polymeric inclusions turns out to be crucial to achieve good confinement of nonleaky modes, although it is accompanied by an increase in dissipation. It should be emphasized that our demonstration of trapped-mode zero and negative group velocity near Scholte-Stoneley band gaps provides a basis on which to construct a Scholte-Stoneley acoustic microscopy, ${ }^{30}$ using flat lenses and near field coupling strategies instead of concave acoustic lenses.

\section{ACKNOWLEDGMENTS}

This work has been supported in part by the Spanish MICINN (MAT2010-16879, MAT2010-14885, Consolider CSD2007-00046, and NanoLight.es), the EU (NMP4-SL2008-213669-ENSEMBLE), Generalitat Valenciana (PROMETEO 2010/043), and Universidad Politecnica de Valencia (PAID-06-10-1839). H.E. acknowledges financial support through JAE from CSIC. We thank B. Bonello for fruitful discussions. We gratefully acknowledge help from J. A. GarcíaManrique, J. V. Giner, and I. Rodríguez in PP fabrication.

\footnotetext{
*hector.estrada@icmm.csic.es

${ }^{\dagger}$ fmese@fis.upv.es

${ }^{1}$ J. Dintinger, S. Klein, and T. Ebbesen, Adv. Mater. 18, 1267 (2006).

${ }^{2}$ T. V. Teperik, F. J. Garcia de Abajo, A. G. Borisov, M. Abdelsalam, P. N. Bartlett, Y. Sugawara, and J. J. Baumberg, Nat. Photon. 2, 299 (2008).

${ }^{3}$ H. Estrada, P. Candelas, A. Uris, F. Belmar, F. J. García de Abajo, and F. Meseguer, Phys. Rev. Lett. 101, 084302 (2008).

${ }^{4}$ T. W. Ebbesen, H. J. Lezec, H. F. Ghaemi, T. Thio, and P. A. Wolff, Nature (London) 391, 667 (1998).

${ }^{5}$ G. P. Wilson and W. W. Soroka, J. Acoust. Soc. Am. 37, 286 (1965).

${ }^{6}$ F. J. García de Abajo, H. Estrada, and F. J. Meseguer, New J. Phys. 11, 093013 (2009).

${ }^{7}$ H. Estrada, F. J. García de Abajo, P. Candelas, A. Uris, F. Belmar, and F. Meseguer, Phys. Rev. Lett. 102, 144301 (2009).

${ }^{8}$ L. Rayleigh, Proc. London Math. Soc. 17, 4 (1885).

${ }^{9}$ H. Lamb, Proc. R. Soc. A 93, 114 (1917).
}

${ }^{10}$ J. G. Scholte, Proc. Kon. Noderl. Akad. Wetensch. 45, 159 (1942).

${ }^{11}$ L. Kelders, J. F. Allard, and W. Lauriks, J. Acoust. Soc. Am. 103, 2730 (1998).

${ }^{12}$ Z. He, H. Jia, C. Qiu, Y. Ye, R. Hao, M. Ke, and Z. Liu, Phys. Rev. B 83, 132101 (2011).

${ }^{13}$ J. Christensen, L. Martin-Moreno, and F. J. Garcia-Vidal, Phys. Rev. Lett. 101, 014301 (2008).

${ }^{14}$ L. Zhou and G. A. Kriegsmann, J. Acoust. Soc. Am. 121, 3288 (2007).

${ }^{15}$ M.-H. Lu, X.-K. Liu, L. Feng, J. Li, C.-P. Huang, Y.-F. Chen, Y.-Y. Zhu, S.-N. Zhu, and N.-B. Ming, Phys. Rev. Lett. 99, 174301 (2007).

${ }^{16}$ B. Hou, J. Mei, M. Ke, W. Wen, Z. Liu, J. Shi, and P. Sheng, Phys. Rev. B 76, 054303 (2007).

${ }^{17}$ J. Christensen, A. I. Fernandez-Dominguez, de F. Leon-Perez, L. Martin-Moreno, and F. J. Garcia-Vidal, Nat. Phys. 3, 851 (2007). 
${ }^{18}$ M. A. Breazeale and M. A. Torbett, Appl. Phys. Lett. 29, 456 (1976).

${ }^{19}$ N. F. Declercq, J. Degrieck, R. Briers, and O. Leroy, J. Appl. Phys. 96, 6869 (2004).

${ }^{20}$ Z. Liang and J. Li, AIP Advances 1, 041503 (2011).

${ }^{21}$ J.-C. Hsu and T.-T. Wu, Phys. Rev. B 74, 144303 (2006).

${ }^{22}$ S. Mohammadi, A. A. Eftekhar, A. Khelif, W. D. Hunt, and A. Adibi, Appl. Phys. Lett. 92, 221905 (2008).

${ }^{23}$ A. G. Every, R. E. Vines, and J. P. Wolfe, Phys. Rev. B 60, 11755 (1999).

${ }^{24}$ F. J. García de Abajo, H. Estrada, and F. Meseguer (unpublished).

${ }^{25} \rho=1000 \quad\left(\mathrm{~kg} / \mathrm{m}^{3}\right)$ and $c_{\ell}=1480(\mathrm{~m} / \mathrm{s})$ for water; $\rho=$ $2700\left(\mathrm{~kg} / \mathrm{m}^{3}\right), c_{\ell}=6467(\mathrm{~m} / \mathrm{s})$, and $c_{t}=3120(\mathrm{~m} / \mathrm{s})$ for aluminum; $\rho=1270\left(\mathrm{~kg} / \mathrm{m}^{3}\right), c_{\ell}=2770(\mathrm{~m} / \mathrm{s})$, and $c_{t}=1430(\mathrm{~m} / \mathrm{s})$ for PMMA; $\rho=880\left(\mathrm{~kg} / \mathrm{m}^{3}\right), c_{\ell}=2038\left(1+1.8 \times 10^{-4} \mathrm{i}\right)(\mathrm{m} / \mathrm{s})$, and $c_{t}=942\left(1+2.2 \times 10^{-4} \mathrm{i}\right)(\mathrm{m} / \mathrm{s})$ for epoxy resin.

${ }^{26}$ X. Jia, J. Acoust. Soc. Am. 101, 834 (1997).

${ }^{27}$ I. Núñez, R. K. Ing, C. Negreira, and M. Fink, J. Acoust. Soc. Am. 107, 2370 (2000).

${ }^{28}$ D. Alleyne and P. Cawley, J. Acoust. Soc. Am. 89, 1159 (1991).

${ }^{29}$ C. Glorieux, K. V. de Rostyne, K. Nelson, W. Gao, W. Lauriks, and J. Thoen, J. Acoust. Soc. Am. 110, 1299 (2001).

${ }^{30}$ R. G. Maev, Acoustic Microscopy (Wiley-VCH Verlag GmbH \& Co. Weinheim, 2008).

${ }^{31}$ See Supplemental Material at http://link.aps.org/supplemental/ 10.1103/PhysRevB.85.174301 for animated versions of Fig. 3 panels. 